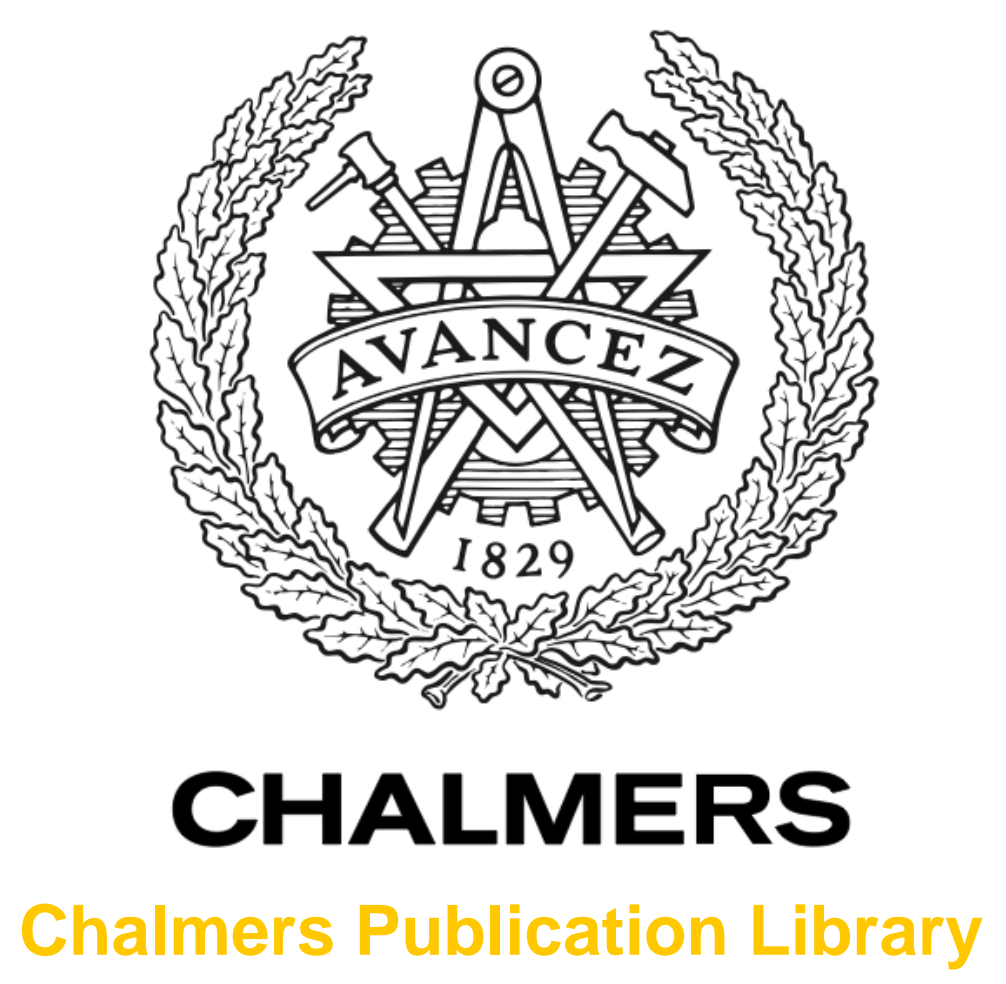

On clock offset and skew estimation with exponentially distributed delays

This document has been downloaded from Chalmers Publication Library (CPL). It is the author's version of a work that was accepted for publication in:

IEEE International Conference on Communications (ISSN: 1550-3607)

Citation for the published paper:

Wanlu, S. ; Brännström, F. ; Ström, E. (2013) "On clock offset and skew estimation with exponentially distributed delays". IEEE International Conference on Communications pp. 1872-1877.

http://dx.doi.org/10.1109/ICC.2013.6654794

Downloaded from: http://publications.lib.chalmers.se/publication/192462

Notice: Changes introduced as a result of publishing processes such as copy-editing and formatting may not be reflected in this document. For a definitive version of this work, please refer to the published source. Please note that access to the published version might require a subscription.

Chalmers Publication Library (CPL) offers the possibility of retrieving research publications produced at Chalmers University of Technology. It covers all types of publications: articles, dissertations, licentiate theses, masters theses, conference papers, reports etc. Since 2006 it is the official tool for Chalmers official publication statistics. To ensure that Chalmers research results are disseminated as widely as possible, an Open Access Policy has been adopted.

The CPL service is administrated and maintained by Chalmers Library. 


\title{
On Clock Offset and Skew Estimation with Exponentially Distributed Delays
}

\author{
Wanlu Sun, Fredrik Brännström, and Erik G. Ström \\ Department of Signals and Systems, Chalmers University of Technology, Gothenburg, Sweden \\ \{wanlu, fredrik.brannstrom, erik.strom\}@chalmers.se
}

\begin{abstract}
This paper investigates the problem of clock synchronization of nodes in a wireless sensor network based on the two-way timing message exchange mechanism with an unknown deterministic transmission delay and random exponential transmission delays. Without knowing the fixed delay, a novel synchronization scheme is proposed for the linear clock model, which works well in both symmetric and asymmetric links. In the proposed algorithm, the clock skew and offset are estimated by directly utilizing mean square error as the metric to be optimized. This consideration results in significant performance improvements compared to the existing synchronization methods, especially for a small number of observations and large standard deviation of the random delays.
\end{abstract}

Index Terms-Wireless sensor network, clock synchronization, clock skew, exponential random delays.

\section{INTRODUCTION}

Recently, wireless sensor networks (WSNs) have emerged as an interesting and important research area. As WSNs consist of many small scale devices and all the devices run their own clocks, clock synchronization becomes critical for efficient applications of WSNs.

Existing studies on clock synchronization mainly focus on the protocol design. However, the clock synchronization problem is inherently related to parameter estimation. The performance of synchronization can be improved by adopting a statistical signal processing framework [1].

Here we consider a simple network comprised by two nodes, $A$ and $B$, with imperfect clocks. The nodes exchange a number of time stamps over a channel with random delays, and the data collected at Node $B$ is used to estimate the clock values of Node $A$. Note that even though this paper considers the synchronization between a pair of nodes, extension to networkwide synchronization can be directly achieved by the combination of building a hierarchical structure and synchronizing two nodes with adjacent levels.

In practice, the challenges of clock synchronization are mainly twofold. Firstly, in the absence of delay during the transmission between Node $B$ and Node $A$, Node $B$ will immediately know the relative clock value difference with

This work has been supported in part by SAFER-Vehicle and Traffic Safety Centre, Project A19. Part of this work has been performed in the framework of the FP7 project ICT-317669 METIS, which is partly funded by the EU. The authors would like to acknowledge the contributions of their colleagues in METIS, although the views expressed are those of the authors and do not necessarily represent the project. respect to Node $A$. Nonetheless, in practical wireless networks, various delays have effects on the time stamp exchange procedure, which will complicate the clock synchronization. These delays can be grouped into two parts: fixed delays and random delays. According to different applications and justifications, the random delays are modeled as random variables with different probability density functions (pdfs) (e.g., Gaussian, exponential, Gamma and so forth). As pointed out in [2] [3], in many cases, a single-server $\mathrm{M} / \mathrm{M} / 1$ queue can fittingly represent the cumulative link delay between two nodes, where the random delays are independent and exponentially distributed.

Secondly, the imperfect oscillators will cause the clocks to run at different frequencies. If we only adjust the clock offset but not the skew, the synchronized status can be maintained merely for a short time period. To achieve more accurate synchronization as well as reduce the re-synchronization interval (which could bring energy savings), both offset and skew adjustment should be taken into account. To deal with this problem, authors in [4] and [5] propose joint maximum likelihood (ML) estimation for symmetric Gaussian and exponential random delays, respectively. More recently, without the assumption on the distribution of delays, a synchronization method is presented in [6] to handle asymmetric links as well.

In this paper, under the assumption of exponential random delays and unknown fixed delays, we propose a novel synchronization algorithm for the widely used linear clock relationship model. By directly utilizing mean square error (MSE) as the metric to be optimized, the proposed scheme improves the precision of offset and skew estimation, and thus the corresponding synchronization performance, especially for small number of observations or random delays with large standard deviation. Last but not least, in the proposed scheme, the main computational workload lies in the simple bisection step, which guarantees its moderate complexity and feasibility in practice.

Notation: Uppercase letters stand for random variables, and the corresponding lowercase letters indicate their realizations. Furthermore, boldface letters designate row vectors. For any vector $\mathbf{a}, a_{i}$ denotes the $i$-th element of $\mathbf{a}$. 


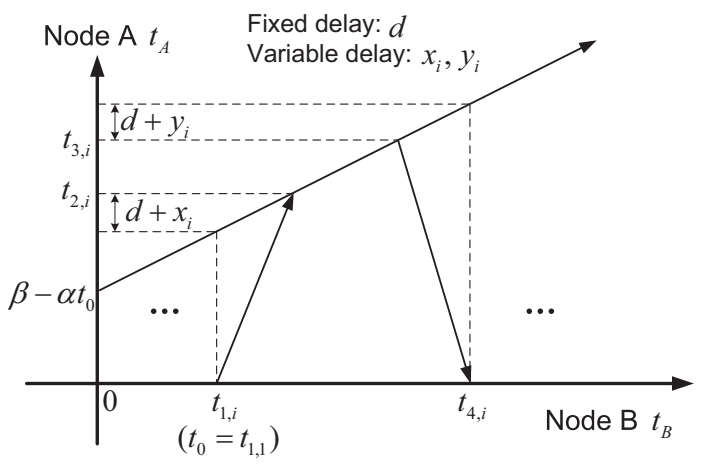

Figure 1. Two-way timing message exchange between nodes $B$ and $A$.

\section{SySTEM MODEL}

\section{A. Clock Value Relationship Model}

Assume Node $B$ (with clock value $t_{B}$ ) needs to synchronize to Node $A$ (with clock value $t_{A}$ ). The clock value relationship between the two nodes is represented as

$$
t_{A}=t_{B}+\alpha\left(t_{B}-t_{0}\right)+\beta,
$$

where $\alpha$ denotes the relative skew, and $\beta$ indicates the relative offset at clock $B$ 's time $t_{0}$, respectively. We model $\{\alpha, \beta\}$ as unknown deterministic parameters. Even though there are only two nodes in our model, the synchronization scheme could be used for the network-side synchronization, where $\{\alpha, \beta\}$ varies among different pairs of nodes. Thereby, when the synchronization algorithm is designed between two nodes, it should also work well for other pairs in the network. Thus the worst case consideration in the algorithm design is more reasonable to guarantee the synchronization between any two nodes in the entire network. Fortunately, in practice, according to the prior knowledge of clock qualities, which could be found in the specifications, we can have some range constraints on $\alpha$, i.e., $|\alpha| \leq L_{\alpha}$. Based on the estimation of $\{\alpha, \beta\}$, Node $B$ can estimate Node $A$ 's clock value as

$$
\hat{t}_{A} \triangleq t_{B}+\hat{\alpha}\left(t_{B}-t_{0}\right)+\hat{\beta} .
$$

\section{B. Time Stamp Exchange Model}

Fig. 1 shows a mechanism of a two-way timing message exchange [7] between Node $B$ and Node $A$, where the timing messages are assumed to be exchanged $N$ times. In the $i$-th round of exchange, Node $B$ records its current clock value as the time stamp $t_{1, i}$ and sends a message to Node $A$ at the same time. Node $A$ records its clock value $t_{2, i}$ at the reception of that message. Then Node $A$ sends at $t_{3, i}$ another message containing $t_{2, i}$ and $t_{3, i}$ to Node $B$. Finally, Node $B$ records its clock value as $t_{4, i}$ when receiving the message. Note that $t_{1, i}$ and $t_{4, i}$ are the time stamps provided by Node $B$ 's clock, while $t_{2, i}$ and $t_{3, i}$ are the time stamps recorded by Node $A$ 's clock. Therefore, after $N$ rounds of message exchanges, Node $B$ has the access to a set of time stamps $\left\{\mathbf{t}_{1}, \mathbf{t}_{2}, \mathbf{t}_{3}, \mathbf{t}_{4}\right\}=\left\{\left[t_{1, i}\right]_{i=1}^{N},\left[t_{2, i}\right]_{i=1}^{N},\left[t_{3, i}\right]_{i=1}^{N},\left[t_{4, i}\right]_{i=1}^{N}\right\}$. Let $t_{0}$ be the clock value of Node $B$ when it sends the first message, i.e., $t_{0}=t_{1,1}$.

Based on (1) and Fig. 1, the clock values at $t_{1, i}$ and $t_{4, i}$ at Node $A$ can be expressed as

$$
\begin{aligned}
& t_{2, i}=t_{1, i}+\alpha\left(t_{1, i}-t_{0}\right)+\beta+d+x_{i}, \quad i=1,2, \ldots, N, \\
& t_{3, i}=t_{4, i}+\alpha\left(t_{4, i}-t_{0}\right)+\beta-d-y_{i}, \quad i=1,2, \ldots, N,
\end{aligned}
$$

where $d$ denotes the fixed delay, $x_{i}$ and $y_{i}$ represent the realizations of random delays in the transmissions from Node $B$ to Node $A$ and from Node $A$ to Node $B$, respectively. These delays are in reference to Node $A$ 's clock. Here $\left\{X_{i}\right\}_{i=1}^{N}$ and $\left\{Y_{i}\right\}_{i=1}^{N}$ are modeled as independent exponentially distributed random variables with mean values (or standard deviation) $\lambda_{X}$ and $\lambda_{Y}$, respectively. It is not necessary that $\lambda_{X}=\lambda_{Y}$, i.e., our system model covers both symmetric and asymmetric links. Moreover, we can assume that the elements of $\mathbf{T}_{2}$ and $\mathbf{T}_{3}$, i.e., the random vectors whose outcomes are $\mathbf{t}_{2}$ and $\mathbf{t}_{3}$, are independent random variables as long as the interval of different rounds is long enough compared to $\lambda_{X}$ and $\lambda_{Y}$. Now the task is to estimate $\{\alpha, \beta\}$ for the given model (3) and (4), and then synchronize Node $B$ 's clock to Node $A$ 's via (2).

\section{Proposed Clock Synchronization Algorithm}

In the class of unbiased estimators, the minimum variance unbiased (MVU) estimator has the smallest variance. When there is only clock offset, [8] derives the MVU estimator for the clock offset. On the other hand, when the clock skew exists as well, the joint MVU estimator of clock offset and skew has not been found so far. Instead, [5] presents the ML estimator for this joint estimation. The ML estimation has nice properties in the presence of a large number of observations (i.e., unbiasedness, asymptotic efficiency and consistence) [9]. Nevertheless, a more desirable estimator is the one with lower MSE, which is a direct measure of estimation error. Furthermore, from the perspective of energy saving and fast convergence, the methodology that can achieve synchronization at the price of low number of observations is more valuable. Therefore, in this paper, we formulate an alternative scheme considering MSE criterion for exponentially distributed random delays, which decreases MSE of the parameter estimation compared to existing synchronization methods, especially for small $N$ or large $\lambda_{X}$ and $\lambda_{Y}$.

For the models in (3) and (4), and under the assumption of independence of $\left\{T_{2, i}\right\}$ and $\left\{T_{3, i}\right\}$ for $i=1,2, \ldots, N$, the pdfs $p_{\mathbf{T}_{2}}\left(\mathbf{t}_{2} ; \alpha, \beta\right)$ and $p_{\mathbf{T}_{3}}\left(\mathbf{t}_{3} ; \alpha, \beta\right)$ are given by

$$
\begin{aligned}
p_{\mathbf{T}_{2}}\left(\mathbf{t}_{2} ; \alpha, \beta\right) & \frac{1}{\lambda_{x}^{N}} \exp \left\{-\frac{1}{\lambda_{x}} \sum_{i=1}^{N}\left(t_{2, i}-t_{1, i}-\alpha\left(t_{1, i}-t_{0}\right)-\beta-d\right)\right\} \\
& \cdot \prod_{i=1}^{N} u\left(t_{2, i}-t_{1, i}-\alpha\left(t_{1, i}-t_{0}\right)-\beta-d\right)
\end{aligned}
$$


and

$$
\begin{aligned}
p_{\mathbf{T}_{3}}\left(\mathbf{t}_{3} ; \alpha, \beta\right) & =\frac{1}{\lambda_{y}^{N}} \exp \left\{-\frac{1}{\lambda_{y}} \sum_{i=1}^{N}\left(t_{4, i}-t_{3, i}+\alpha\left(t_{4, i}-t_{0}\right)+\beta-d\right)\right\} \\
& \cdot \prod_{i=1}^{N} u\left(t_{4, i}-t_{3, i}+\alpha\left(t_{4, i}-t_{0}\right)+\beta-d\right)
\end{aligned}
$$

respectively, where $u(\cdot)$ denotes the unit step function.

In the proposed algorithm, we estimate $\{\alpha, \beta\}$ for the models in (3) and (4) separately, and then average the results. The estimators based on (3) and (4) are denoted by $\left\{\hat{\alpha}_{1}, \hat{\beta}_{1}\right\}$ and $\left\{\hat{\alpha}_{2}, \hat{\beta}_{2}\right\}$, respectively. Due to the similarity of the estimation procedures for (3) and (4), only the derivations for the model in (3) will be presented.

Since the given pdf in (5) cannot be factorized to find a sufficient statistic based on the Neyman-Fisher Factorization (NFF) theorem [9] and the Rao-Blackwell-Lehmann-Schefe (RBLS) theorem [9], it is not easy to deduce the joint MVU estimator for $\{\alpha, \beta\}$. Meanwhile, since our final aim is to minimize MSE rather than variance, if possible, we would like to construct biased estimators instead of the MVU estimator to further decrease the MSE. As $\{\alpha, \beta\}$ are deterministic parameters, the minimum mean square error (MMSE) estimation of $\{\alpha, \beta\}$ will in general depend on the unknown $\{\alpha, \beta\}$ themselves and therefore not realizable. Moreover, to simplify the analysis, we may restrict attention to the estimators with linear bias [10]. In other words, under the assumption that the MVU estimator $\hat{\alpha}_{1 \mathrm{U}}$ for $\alpha$ and the associated variance $\sigma_{\hat{\alpha}_{1 \mathrm{U}}}^{2}$, as well as the MVU estimator $\hat{\beta}_{1 \mathrm{U}}$ for $\beta$ and the associated variance $\sigma_{\hat{\beta}_{1 \mathrm{U}}}^{2}$ are available, we will try to find the biased estimators $\hat{\alpha}_{1}=\left(1+\eta_{\alpha}\right) \hat{\alpha}_{1 \mathrm{U}}$ and $\hat{\beta}_{1}=\left(1+\eta_{\beta}\right) \hat{\beta}_{1 \mathrm{U}}$, where $\eta_{\alpha} \hat{\alpha}_{1 \mathrm{U}}$ and $\eta_{\beta} \hat{\beta}_{1 \mathrm{U}}$ will determine the biases. At the same time, as mentioned in Section II, the estimation of $\{\alpha, \beta\}$ is supposed to achieve the synchronization of any pair in the entire network, and there will always be limitations imposed on $\alpha\left(|\alpha| \leq L_{\alpha}\right)$. Due to aforementioned reasons, we define our problem as a minimax optimization problem, which is formulated as

$$
\begin{aligned}
& \min _{\eta_{\alpha}, \eta_{\beta}} \max _{|\alpha| \leq L_{\alpha}}\left\{\operatorname{MSE}\left(\left[\hat{\alpha}_{1}, \hat{\beta}_{1}\right]\right)\right\} \\
& \quad=\min _{\eta_{\alpha}} \max _{|\alpha| \leq L_{\alpha}}\left\{\operatorname{MSE}\left(\hat{\alpha}_{1}\right)\right\}+\min _{\eta_{\beta}}\left\{\operatorname{MSE}\left(\hat{\beta}_{1}\right)\right\} .
\end{aligned}
$$

The optimum of problem (7) does not depend on $\alpha$ (we will prove this later). On the other hand, this optimum is a function of $\beta$, which means we can not find a linear biased estimator $\hat{\beta}_{1}$ which gives lower MSE than $\hat{\beta}_{1 \mathrm{U}}$ for all possible $\beta$. These phenomena come from the fact that only the range constraint on $\alpha$ is available but not that on $\beta$. According to the foregoing analysis, we can make use of three guidelines for acquiring $\left\{\hat{\alpha}_{1}, \hat{\beta}_{1}\right\}$ :

1) estimate $\alpha$ and $\beta$ separately, i.e., estimate one parameter when assuming the other one is known, and then plug the results into each other in a certain sequence which will be explained later;
2) when estimating $\alpha$, introduce linear bias for to decrease MSE by utilizing the available $L_{\alpha}$;

3) when estimating $\beta$, use the MVU estimator since we do not have prior knowledge about $\beta$.

Firstly, suppose $\beta$ is known and $\alpha$ is estimated. Considering the pdf in (5), we can find a sufficient statistic for $\alpha$ as $T\left(\mathbf{t}_{2}\right)=$ $\min _{i \in\{2, \ldots, N\}}\left\{\frac{t_{2, i}-t_{1, i}-\beta-d}{t_{1, i}-t_{0}}\right\}$ based on the NFF theorem [9]. Furthermore, according to the RBLS theorem [9], the MVU estimator of $\alpha$ is calculated as (see Appendix A)

$$
\hat{\alpha}_{1 \mathrm{U}}=\min _{i \in\{2, \ldots, N\}}\left\{\frac{t_{2, i}-t_{1, i}-\beta-d}{t_{1, i}-t_{0}}\right\}-\frac{\lambda_{X}}{\sum_{i=2}^{N}\left(t_{1, i}-t_{0}\right)},
$$

and the variance of $\hat{\alpha}_{1 \mathrm{U}}$ is

$$
\sigma_{\hat{\alpha}_{1 \mathrm{U}}}^{2}=\frac{\lambda_{X}^{2}}{\left(\sum_{i=2}^{N}\left(t_{1, i}-t_{0}\right)\right)^{2}}
$$

Hence, $\eta_{\alpha}$ is derived as

$$
\begin{aligned}
\eta_{\alpha} & =\arg \min _{\eta} \max _{|\alpha| \leq L_{\alpha}}\{\underbrace{(1+\eta)^{2} \sigma_{\hat{\alpha}_{1 U}}^{2}+\eta^{2} \alpha^{2}}_{\operatorname{MSE}\left(\hat{\alpha}_{1}\right)}\} \\
& =\min _{\eta}\left\{(1+\eta)^{2} \sigma_{\hat{\alpha}_{1 \mathrm{U}}}^{2}+\eta^{2} L_{\alpha}^{2}\right\} \\
& =\frac{-\sigma_{\hat{\alpha}_{1 \mathrm{U}}}^{2}}{\sigma_{\hat{\alpha}_{1 \mathrm{U}}}^{2}+L_{\alpha}^{2}},
\end{aligned}
$$

where the second equality follows because $(1+\eta)^{2} \sigma_{\hat{\alpha}_{1 \mathrm{U}}}^{2}+$ $\eta^{2} \alpha^{2}$ is monotonically increasing in $|\alpha|$, and the last equality from the optimality criterion for differentiable functions [11, pp. 623]. Correspondingly,

$$
\hat{\alpha}_{1}=\left(1+\eta_{\alpha}\right) \hat{\alpha}_{1 \mathrm{U}}=\frac{L_{\alpha}^{2}}{L_{\alpha}^{2}+\sigma_{\hat{\alpha}_{1 \mathrm{U}}}^{2}} \hat{\alpha}_{1 \mathrm{U}}
$$

Besides, $\operatorname{MSE}\left(\hat{\alpha}_{1}\right)$ is calculated as

$$
\begin{aligned}
& \operatorname{MSE}\left(\hat{\alpha}_{1}\right)=\left(\frac{L_{\alpha}^{2}}{\sigma_{\hat{\alpha}_{1 \mathrm{U}}}^{2}+L_{\alpha}^{2}}\right)^{2} \sigma_{\hat{\alpha}_{1 \mathrm{U}}}^{2}+\left(\frac{\sigma_{\hat{\alpha}_{1 \mathrm{U}}}^{2}}{\sigma_{\hat{\alpha}_{1 \mathrm{U}}}^{2}+L_{\alpha}^{2}}\right)^{2} \alpha^{2} \\
& \leq\left(\frac{L_{\alpha}^{2}}{\sigma_{\hat{\alpha}_{1 \mathrm{U}}}^{2}+L_{\alpha}^{2}}\right)^{2} \sigma_{\hat{\alpha}_{1 \mathrm{U}}}^{2}+\left(\frac{\sigma_{\hat{\alpha}_{1 \mathrm{U}}}^{2}}{\sigma_{\hat{\alpha}_{1 \mathrm{U}}}^{2}+L_{\alpha}^{2}}\right)^{2} L_{\alpha}^{2} \\
& =\frac{L_{\alpha}^{2}}{L_{\alpha}^{2}+\sigma_{\hat{\alpha}_{1 \mathrm{U}}}^{2}} \sigma_{\hat{\alpha}_{1 \mathrm{U}}}^{2} \leq \sigma_{\hat{\alpha}_{1 \mathrm{U}}}^{2}=\operatorname{MSE}\left(\hat{\alpha}_{1 \mathrm{U}}\right)
\end{aligned}
$$

which shows that $\operatorname{MSE}\left(\hat{\alpha}_{1}\right) \leq \operatorname{MSE}\left(\hat{\alpha}_{1 \mathrm{U}}\right)$ for all $|\alpha| \leq L_{\alpha}$.

On the other hand, assume $\alpha$ is known and we want to estimate $\beta$. The component $\min _{\eta_{\beta}} \operatorname{MSE}\left(\hat{\beta}_{1}\right)$ of problem (7) depends on the actual $\beta$ value, which means that the lack of constraint on $\beta$ limits the further improvement of the linear biased estimator compared to the MVU estimator. In this case, we can simply set $\eta_{\beta}=0$ and thus $\hat{\beta}_{1}=\hat{\beta}_{1 \mathrm{U}}$. Using a similar approach as in the derivation of $\hat{\alpha}_{1 \mathrm{U}}$ in Appendix $\mathrm{A}, \hat{\beta}_{1}$ is derived as

$$
\hat{\beta}_{1}=\hat{\beta}_{1 \mathrm{U}}=\min _{i}\left\{t_{2, i}-t_{1, i}-\alpha\left(t_{1, i}-t_{0}\right)\right\}-d-\frac{\lambda_{X}}{N}
$$




$$
\begin{gathered}
\hat{\alpha}_{1}=\frac{L_{\alpha}^{2}}{L_{\alpha}^{2}+\sigma_{\hat{\alpha}_{1 \mathrm{U}}}^{2}}\left\{\min _{i \in\{2, \ldots, N\}}\left\{\frac{t_{2, i}-t_{1, i}-\min _{i}\left\{t_{2, i}-t_{1, i}-\hat{\alpha}_{1}\left(t_{1, i}-t_{0}\right)\right\}+\frac{\lambda_{X}}{N}}{t_{1, i}-t_{0}}\right\}-\frac{\lambda_{X}}{\sum_{i=2}^{N}\left(t_{1 i}-t_{0}\right)}\right\} \\
\hat{\alpha}_{2}=\frac{L_{\alpha}^{2}}{L_{\alpha}^{2}+\sigma_{\hat{\alpha}_{2 \mathrm{U}}}^{2}}\left\{-\min _{i}\left\{\frac{t_{4, i}-t_{3, i}-\min _{i}\left\{t_{4, i}-t_{3, i}+\hat{\alpha}_{2}\left(t_{4, i}-t_{0}\right)\right\}+\frac{\lambda_{Y}}{N}}{\left(t_{4, i}-t_{0}\right)}\right\}+\frac{\lambda_{Y}}{\sum_{i=1}^{N}\left(t_{4 i}-t_{0}\right)}\right\}
\end{gathered}
$$

with

$$
\operatorname{MSE}\left(\hat{\beta}_{1}\right)=\sigma_{\hat{\beta}_{1 \mathrm{U}}}^{2}=\frac{\lambda_{X}^{2}}{N^{2}} .
$$

From (8), (11), and (13), we observe that the estimators $\hat{\alpha}_{1}$ and $\hat{\beta}_{1}$ are functions of each other. By plugging the expression of $\hat{\beta}_{1}$ into that of $\hat{\alpha}_{1}$, the non-linear equation (15) for $\hat{\alpha}_{1}$ is obtained, shown on top of next page. Note that $\hat{\alpha}_{1}$ is independent of the fixed delay $d$. In our experience, the bisection method [11] is an efficient scheme to solve (15) for $\hat{\alpha}_{1}$. In the next step, $\hat{\alpha}_{1}$ is put back into (13), and $\hat{\beta}_{1}$ can be calculated directly. This concludes the estimation of $\{\alpha, \beta\}$ for the model (3).

The above procedure can also be applied to the model in (4) and the associated pdf $p_{\mathbf{T}_{3}}\left(\mathbf{t}_{3} ; \alpha, \beta\right)$ in (6). The results are shown in equation (16) (on top of this page) for estimating $\hat{\alpha}_{2}$ and equations (17)--(20) as follows

$$
\begin{gathered}
\operatorname{MSE}\left(\hat{\alpha}_{2}\right)=\left(\frac{L_{\alpha}^{2}}{\sigma_{\hat{\alpha}_{2 \mathrm{U}}}^{2}+L_{\alpha}^{2}}\right)^{2} \sigma_{\hat{\alpha}_{2 \mathrm{U}}}^{2}+\left(\frac{\sigma_{\hat{\alpha}_{2 \mathrm{U}}}^{2}}{\sigma_{\hat{\alpha}_{2 \mathrm{U}}}^{2}+L_{\alpha}^{2}}\right)^{2} \alpha^{2},(17) \\
\hat{\beta}_{2}=-\min _{i}\left\{t_{4, i}-t_{3, i}+\alpha\left(t_{4, i}-t_{0}\right)\right\}+d+\frac{\lambda_{Y}}{N},
\end{gathered}
$$

and

$$
\operatorname{MSE}\left(\hat{\beta}_{2}\right)=\sigma_{\hat{\beta}_{2 \mathrm{U}}}^{2}=\frac{\lambda_{Y}^{2}}{N^{2}}
$$

where

$$
\sigma_{\hat{\alpha}_{2 \mathrm{U}}}^{2}=\frac{\lambda_{Y}}{\left(\sum_{i=1}^{N}\left(t_{4, i}-t_{0}\right)\right)^{2}} .
$$

Finally, we can average the results from models (3) and (4), and obtain $\{\hat{\alpha}, \hat{\beta}\}$ as

$$
\hat{\alpha}=\frac{\hat{\alpha}_{1}+\hat{\alpha}_{2}}{2}, \quad \hat{\beta}=\frac{\hat{\beta}_{1}+\hat{\beta}_{2}}{2} .
$$

The root mean square error (RMSE) of $\{\hat{\alpha}, \hat{\beta}\}$ are, assuming independent estimates,

$$
\begin{gathered}
\operatorname{RMSE}(\hat{\alpha})=\sqrt{\frac{\operatorname{MSE}\left(\hat{\alpha}_{1}\right)+\operatorname{MSE}\left(\hat{\alpha}_{2}\right)}{4}+\frac{\mathbb{B}\left(\hat{\alpha}_{1}\right) \mathbb{B}\left(\hat{\alpha}_{2}\right)}{2}}, \\
\operatorname{RMSE}(\hat{\beta})=\sqrt{\frac{\operatorname{MSE}\left(\hat{\beta}_{1}\right)+\operatorname{MSE}\left(\hat{\beta}_{2}\right)}{4}}
\end{gathered}
$$

where $\mathbb{B}(\cdot)$ represents the bias of the estimator, and

$$
\mathbb{B}\left(\hat{\alpha}_{1}\right)=\frac{-\sigma_{\hat{\alpha}_{1 U}}^{2}}{\sigma_{\hat{\alpha}_{1 U}}^{2}+L_{\alpha}^{2}} \alpha, \quad \mathbb{B}\left(\hat{\alpha}_{2}\right)=\frac{-\sigma_{\hat{\alpha}_{2 U}}^{2}}{\sigma_{\hat{\alpha}_{2 U}}^{2}+L_{\alpha}^{2}} \alpha .
$$

Note that all MSE calculations in Section III are for one single parameter and under the condition that the other parameter is perfectly known. Because of the averaging in (21), there is no need to know $d$ for the estimation of $\beta$.

Furthermore, the main complexity of the proposed method is in the bisection step when estimating $\alpha$, which will terminate after exact $\left\lceil\log _{2}\left(2 L_{\alpha} / \epsilon\right)\right\rceil$ iterations for the given interval $\left[-L_{\alpha}, L_{\alpha}\right]$ and precision tolerance $\epsilon$ [11]. Through simulation evaluations in Section IV, we could find that the number of iterations is usually less than 10 for reasonable values of $L_{\alpha}$ and $\epsilon$. This observation guarantees the acceptable complexity and feasibility of the proposed synchronization algorithm in practice.

The complete procedures of the proposed synchronization scheme are summarized in Algorithm 1.

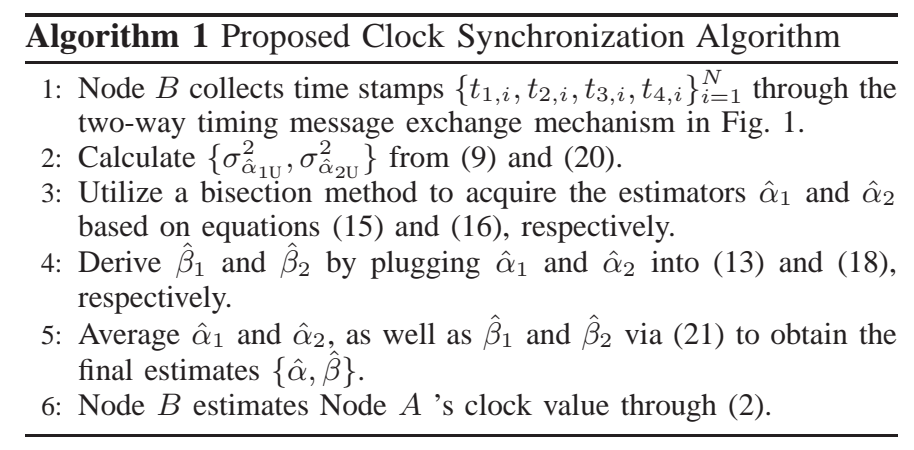

\section{Simulation Results}

In this section, simulation results are presented to compare the performance of the proposed clock synchronization algorithm to the following schemes: 1) Least Square ( $L S)$ estimator in [13]; 2) $M L$ estimator for exponential delays [5]; 3) the scheme described in [6]. The evaluations also include the MVU estimator $\left(\hat{\alpha}_{\mathrm{U}}\right.$ or $\left.\hat{\beta}_{\mathrm{U}}\right)$ for one parameter assuming the other one is perfectly known $(1 d-M V U E)$, i.e.,

$$
\begin{aligned}
& \operatorname{RMSE}\left(\hat{\alpha}_{\mathrm{U}}\right)=0.5 \sqrt{\sigma_{\hat{\alpha}_{1 \mathrm{U}}}^{2}+\sigma_{\hat{\alpha}_{2 \mathrm{U}}}^{2}}, \\
& \operatorname{RMSE}\left(\hat{\beta}_{\mathrm{U}}\right)=0.5 \sqrt{\sigma_{\hat{\beta}_{1 \mathrm{U}}}^{2}+\sigma_{\hat{\beta}_{2 \mathrm{U}}}^{2}} .
\end{aligned}
$$

Likewise, the theoretical RMSE (22) and (23) are contained as well, which represent the performance of the proposed method for one single parameter (1d-Pro). Additionally, as given in [12], we set two different skews $\alpha=10^{-5}$ and $\alpha=10^{-4}$, as well as $L_{\alpha}=2 \times 10^{-4}$ [12] in the experiments. Besides, the interval between the two-way timing messages is assumed to be around 0.1s. Also, we choose the fixed delay as $d=0.001 \mathrm{~s}$, and the offset as $\beta=0.002 \mathrm{~s}$. Finally, in all 


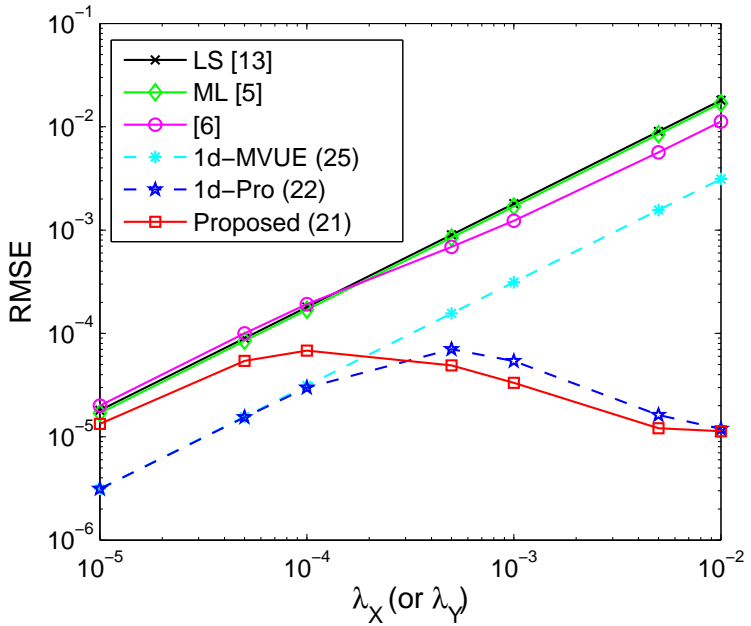

Figure 2. RMSE of the skew $(\alpha)$ estimation versus the standard deviation of the random delays $\left(\lambda_{X}\right.$ or $\left.\lambda_{Y}\right)$ for symmetric links, with $\alpha=10^{-5}$ and $N=6$. The number of bisection iterations is 6 .

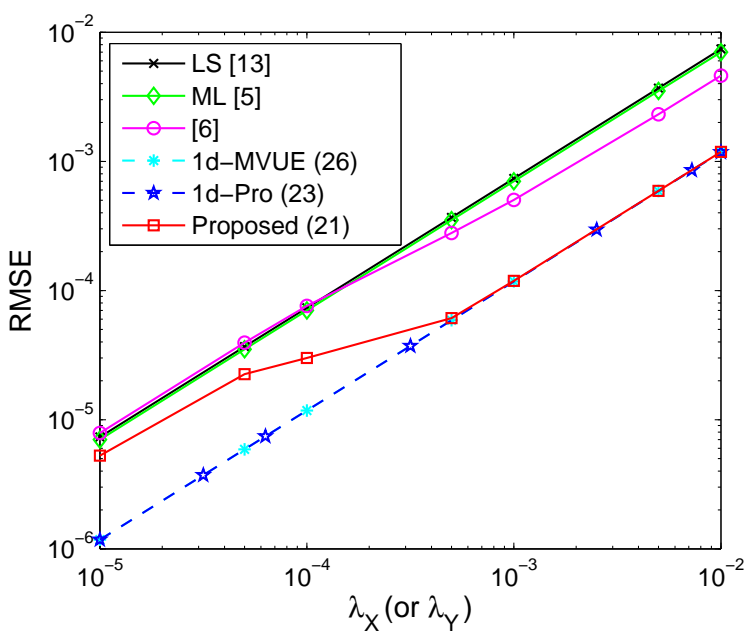

Figure 3. RMSE of the offset $(\beta)$ estimation versus the standard deviation of the random delays $\left(\lambda_{X}\right.$ or $\left.\lambda_{Y}\right)$ for symmetric links, with $\alpha=10^{-5}$ and $N=6$.

simulations, we consider RMSE of the parameter estimators as the performance metric.

Fig. 2 describes the RMSE of the skew estimation versus the standard deviation of the random delays for symmetric links. Firstly, when $\beta$ is available, the theoretical $\operatorname{RMSE}(\hat{\alpha})$ of $1 d$-Pro is smaller than $\operatorname{RMSE}\left(\hat{\alpha}_{\mathrm{U}}\right)$ of $1 d$-MVUE. This phenomenon coincides with the proof in (12). Furthermore, by following the calculation in (22), it could be deduced that $\operatorname{RMSE}(\hat{\alpha})$ is not a monotonic function with respect to $\lambda_{X}$ (or $\left.\lambda_{Y}\right)$, as shown in Fig. 2. Additionally, when $\beta$ is also unknown, the proposed algorithm obviously improves the accuracy of $\alpha$ estimation compared to the existing synchronization schemes, especially when the random delays have relatively large standard deviation. This result guarantees the robustness of the proposed one to different levels of random delays. Besides, it

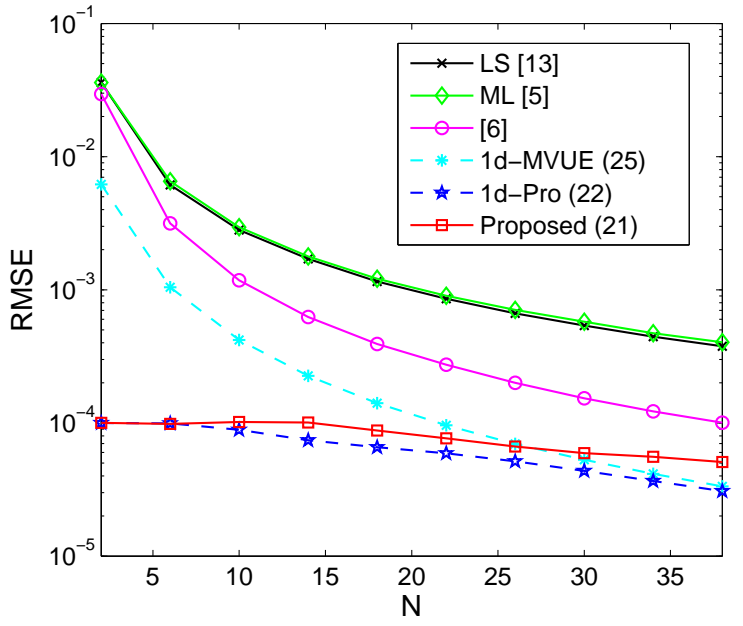

Figure 4. RMSE of the skew ( $\alpha$ ) estimation versus $N$ for asymmetric links, with $\alpha=10^{-4}, \lambda_{X}=0.001$ and $\lambda_{Y}=0.005$. The number of bisection iterations is 6 .

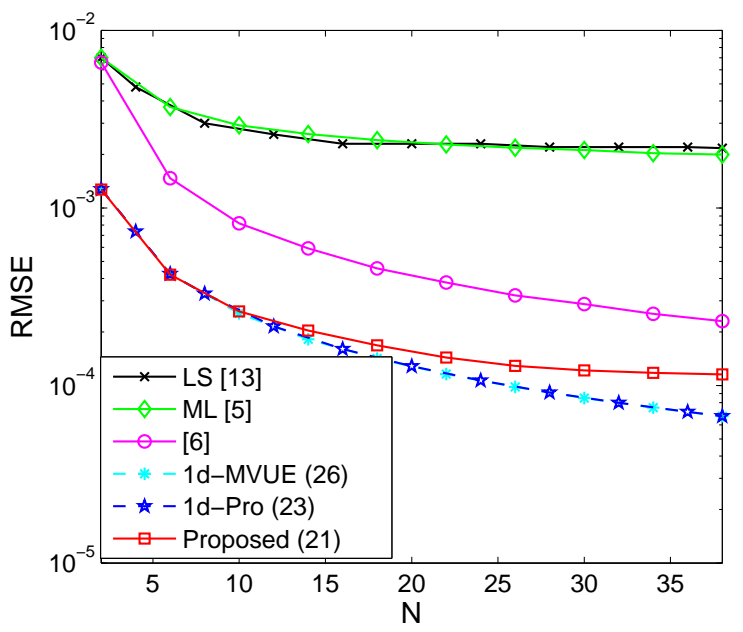

Figure 5. RMSE of offset $(\beta)$ estimation versus $N$ for asymmetric links, with $\alpha=10^{-4}, \lambda_{X}=0.001$ and $\lambda_{Y}=0.005$.

is revealed from Fig. 2 that the RMSE of the proposed method do not monotonically increase with increased $\lambda_{X}$ (or $\lambda_{Y}$ ). This is reasonable since the proposed algorithm, where both $\alpha$ and $\beta$ are estimated, is an extension of $1 d$-Pro. Note that the interesting 'non-monotonic' observation is also found in [14]. On the other hand, even though $\beta$ is available in $1 d$-Pro, it still could happen that the proposed scheme beats $1 d$-Pro in the sense that neither of them is optimal with regard to the RMSE of $\alpha$ estimation.

Fig. 3 illustrates the RMSE of the offset estimation under the same parameter settings as in Fig. 2. Not surprisingly, with known $\alpha$, the theoretical $\operatorname{RMSE}(\hat{\beta})$ of $1 d$-Pro overlaps with the $\operatorname{RMSE}\left(\hat{\beta}_{\mathrm{U}}\right)$ of $1 d-M V U E$ since they are identical. Moreover, as demonstrated in this figure, the proposed scheme exhibits superior performance to $L S, M L$ and the method in [6] with respect to $\beta$ estimation. 
In the environment of asymmetric link delays, the results are illustrated in Fig. 4 to evaluate the RMSE of skew estimation against the number of observations. Since the minimax idea is utilized to further decrease the MSE in the estimation of $\alpha$, the $\operatorname{RMSE}(\hat{\beta})$ of $l d$-Pro is better than the $\operatorname{RMSE}\left(\hat{\beta}_{\mathrm{U}}\right)$ of $1 d-M V U E$ as plotted in Fig. 4. Additionally, as shown in Fig. 4, the improvement of the proposed algorithm is significant compared to other synchronization schemes, especially at the region of low observation numbers. This advantage means that the proposed method can achieve relatively high synchronization precision at the price of less energy consumption. Furthermore, Fig. 5 presents the corresponding results for the estimation of the clock offset $\beta$. It is again verified that the proposed scheme outperforms $L S, M L$ and the method in [6].

\section{CONCLUSION}

In this paper, a clock synchronization scheme is proposed in the presence of exponentially distributed random delays and unknown fixed delays. Through exploiting the prior knowledge about the range constraint on the clock skew, the estimation of skew is performed by optimizing an MSE related minimax problem. Besides, the clock offset is estimated by the MVU estimator. The proposed algorithm exhibits improved performance compared with previously proposed schemes, particularly when the number of observations is limited or the standard deviation of random delays is large. Moreover, the acceptable complexity is guaranteed by the simplicity of the bisection method.

\section{APPENDIX A}

Proof of THE MVU ESTIMATOR $\hat{\alpha}_{1 \mathrm{U}}$ IN (8)

For estimating $\alpha$, the given pdf in (5) can be factorized as,

$$
\begin{aligned}
p_{\mathbf{T}_{2}}\left(\mathbf{t}_{2} ; \alpha, \beta\right) & \underbrace{\frac{1}{\lambda_{X}^{N}} \exp \left\{\frac{\sum_{i=1}^{N}\left(t_{2, i}-t_{1, i}-\beta-d\right)}{-\lambda_{X}}\right\} u\left(t_{2,1}-t_{0}-\beta-d\right)}_{h\left(\mathbf{t}_{2}\right)} \\
& \underbrace{\exp \left\{-\frac{\alpha}{\lambda_{X}} \sum_{i=1}^{N}\left(t_{1, i}-t_{0}\right)\right\} u\left(T\left(\mathbf{t}_{2}\right)-\alpha\right)}_{g(T(\mathbf{t}), \alpha)},
\end{aligned}
$$

where

$$
T\left(\mathbf{t}_{2}\right)=\min _{i \in\{2, \ldots, N\}}\left\{\frac{t_{2, i}-t_{1, i}-\beta-d}{t_{1, i}-t_{0}}\right\} .
$$

is the sufficient statistic for $\alpha$ according to the NFF theorem [9].

Now we will show that this sufficient statistic $T\left(\mathbf{t}_{2}\right)$ is complete. Here we introduce a random variable $\Gamma=T\left(\mathbf{T}_{2}\right)-\alpha$. Through the order statistic property [15], it is known that $\Gamma$ follows an exponential distribution with mean value $\lambda_{\Gamma}=$ $\lambda_{X} /\left(\sum_{i=2}^{N}\left(t_{1 i}-t_{0}\right)\right)$. Suppose that for all $\alpha E\left\{f\left(T\left(\mathbf{T}_{2}\right)\right)\right\}=$ 0, i.e., $E\{f(\Gamma+\alpha)\}=0$, which is equivalent to

$$
\int_{0}^{+\infty} f(\gamma+\alpha) \frac{1}{\lambda_{\Gamma}} \exp \left\{-\frac{\gamma}{\lambda_{\Gamma}}\right\} d \gamma=0
$$

for all $\alpha$. This implies $f(\cdot)=0$ a.e. (almost everywhere). Hence $T\left(\mathbf{t}_{2}\right)$ is complete [9].

Furthermore, based on the RBLS theorem [9], since

$$
E\left\{T\left(\mathbf{t}_{2}\right)-\frac{\lambda_{X}}{\sum_{i=2}^{N}\left(t_{1 i}-t_{0}\right)}\right\}=\alpha,
$$

the MVU estimator of $\alpha$ is therefore calculated as (8).

\section{REFERENCES}

[1] Y. Wu, Q. M. Chaudhari, and E. Serpedin, "Clock synchronization of wireless sensor networks," IEEE Signal Processing Magazine, vol. 28, no. 1, pp. 124-138, Jan. 2011.

[2] C. Bovy, H. Mertodimedjo, G. Hooghiemstra, H. Uijterwaal, and P. Mieghem, "Analysis of end-to-end delay measurements in Internet," in Proc. Passive and Active Measurements Workshop, Mar. 2002, pp. 26-33.

[3] V. Paxson, "On calibrating measurements of packet transit times," in Proc. 7th ACM Sigmetrics Conf., Jun. 1998, pp. 11-21.

[4] K. Noh, Q. M. Chaudhari, E. Serpedin, and B. W. Suter, "Novel clock phase offset and skew estimation using two-way timing message exchanges for wireless sensor networks," IEEE Trans. Comm., vol. 55, no. 4, pp. 766-777, Apr. 2007.

[5] M. Leng and Y. Wu, "On joint synchronization of clock offset and skew for wireless sensor networks under exponential delay," in Proc. IEEE ISCAS, Jun. 2010, pp. 461-464.

[6] Q. M. Chaudhari, "A simple and robust clock synchronization scheme," IEEE Trans. on Comm., vol. 60, no. 2, pp. 328-332, Feb. 2012.

[7] S. Ganeriwal, R. Kumar, and M. B. Srivastava, "Timing-sync protocol for sensor networks," in Proc. SenSys 03, Nov. 2003, pp. 138-149.

[8] Q. M. Chaudhari, E. Serpedin, and K. Qaraqe, "On minimum variance unbiased estimation of clock offset in a two-way message exchange mechanism," IEEE Trans. Inf. Theory, vol. 56, no. 6, pp. 2893-2904, Jun. 2010.

[9] S. M. Kay, Fundamentals of Statistical Signal Processing, Vol.1. Estimation Theory. Englewood Cliffs, NJ: Prentice-Hall, 1993.

[10] Y. C. Eldar, "Rethinking biased estimation: improving maximum likelihood and the Cramer-Rao bound," Foundations and Trends in Signal Processing, vol. 1, no. 4, pp. 305-449, 2008

[11] S. Boyd and L. Vandenberghe, Convex optimization. Cambridge University Press, 2004.

[12] F. Ferrari, A. Meier, and L. Thiele, "Accurate clock models for simulating wireless sensor networks," OMNeT++, Mar. 2010.

[13] M. Maroti, B. Kusy, G. Simon, and A. Ledeczi, "The flooding time synchronization protocol," in Proc. 2004 International Conf. Embedded Networked Sensor Systems, pp. 39-49.

[14] Y. C. Eldar, "Minimax estimation of deterministic parameters in linear models with a random model matrix," IEEE Trans. Signal Processing, vol. 54, no. 2, pp. 601-612, Feb. 2006.

[15] J. S. Huang, "Characterizations of the exponential distribution by order statistics," Journal of Applied Probability, vol. 11, no. 3, pp. 605-608, Sep. 1974. 